\title{
Human Ear recognition Using Geometric Features
}

\author{
Mudit Moghey, Ajinkya R. Ghadge, Sagar J. Dalvi ${ }^{1,2,3,4}$ \\ Department of Computer Engineering, Sinhgad Institute of Technology, Kusgaon, Lonavala, Pune 1,2,3,4 $^{1}$
}

\begin{abstract}
Biometrics is the study of automatic techniques for recognizing human beings based on physical or behavioral traits. To find good biometric features, technique has been researched extensively in recent years. Among several biometric features, ear is quite stable because it does not vary with age and emotion. The ear recognition work depends on ear height, reference line cut points, corresponding angles and inner ear curve. The study is performed on the ear in random orientation and shows a greater accuracy than existing dominant approach. The recognition accuracy is increased by using more training images for database. Face recognition by itself, using the same approach, gave a $63 \%$ rank one recognition rate, but when complimented with ear images in a multimodal system improved to $94 \%$ rank one recognition rate.
\end{abstract}

General Terms: K-nearest Neighbors, Ear Edge Detection, Ear Localization, Feature Extraction, Radon and DCT.

Keywords: Image Processing, ear biometrics, curve fitting, ear orientation, ear height line, reference lines, angles.

\section{INTRODUCTION}

Recognition of individuals based on biometric systems is so far increasing. The biometrics system provides consistent recognition by exploring the physical or behavioral characters of human beings and exceptional among all personnel. In recognition process biometric systems are deploying either enhancing the security, reliability and efficiency of an identification process. Based on exceptionality and steadiness of the biometrics during human's lifespan, it is ease to recognize and authentication. Some of biometric applications like finger prints, face, ear and gait. Ear is possible new class of biometric with enviable features are universality, uniqueness and immovability. Ear has vital features, it is having constant structure which do not change with time period (age). It doesn't change shape with expressions, cosmetics and hair dressing.

In past years, many approaches have been developed for ear recognition from $2 \mathrm{D}$ and $3 \mathrm{D}$ images $[1,5,6]$. Ajay Kumar $\mathrm{n}$ and Chenye Wu, worked on 2D images with segmentation and log-gabor filter method [2]. R. Davies and A.S. Mian, given an approach based on 3D local surface features which gives automatic ear recognition [3]. lu lu and zhang xiaoxun proposed statistical shape model to identify ear [4]. Arun Ross, Ayman Abaza based on using ear prints [7]. Syed M, S Islam and Rowan Davies approach on 3D local feature extraction [10]. Anam Tariq and M Usman Akram used preprocessing, haar wavelet and fast normalized cross correlation methods [11]. Xin Dong and Yin Guo, using SIFT keypoint matching method [13]. Lxu chao and tian ying used on fusion Harris mechanism and sift method to recognize [14]. Dasari Naga Shailaja proposed a Geometric approach for ear recognition [16]. Surya prakash and Phalguni gupta used invariant to illumination and pose [17].

Ear recognition technology is useful tool in the biometric systems. Ear has inner and outer boundaries to recognize ear and also having many other sensitive parts as shown in

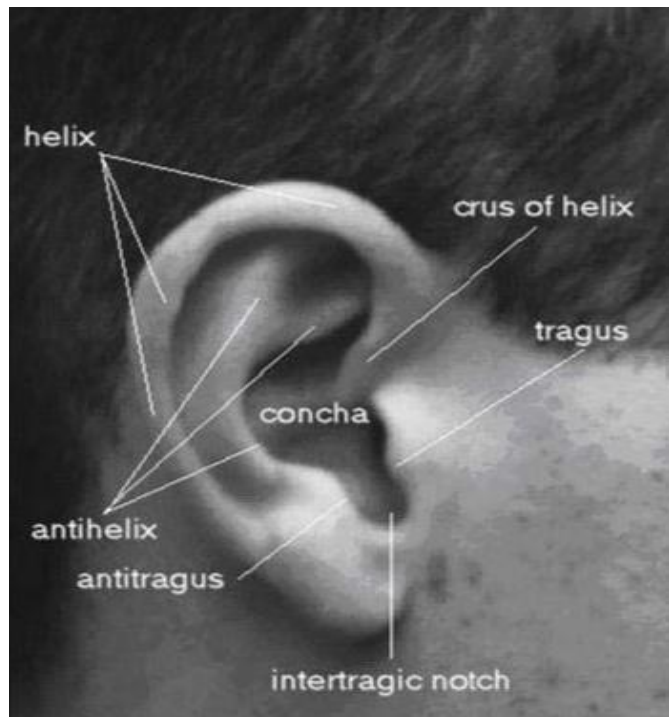

Fig. 1 Ear Edges Image

In this paper, we present canny edge detection method to recognize the ear inner and outer boundaries and removing the noisy data from the captured ear image. To match the input edge image with the database, we present KNN algorithm to vary the exact image for the given input data. Making input edge image as centric and find the related or nearest edge image from the Database. Using centric method it matches the images based on the inner and outer edge locations from the ear images. It gives effective toning technique to identify the edges and it increase the accuracy of ear recognition. Database is having the all the images with their respective inner and outer edge locations in the form of images. The Fig 1.1 shows the structure, shapes and depressions of ear. Ear recognition gives effective and uniqueness to authenticate the person without any changes in future Fig 1. 


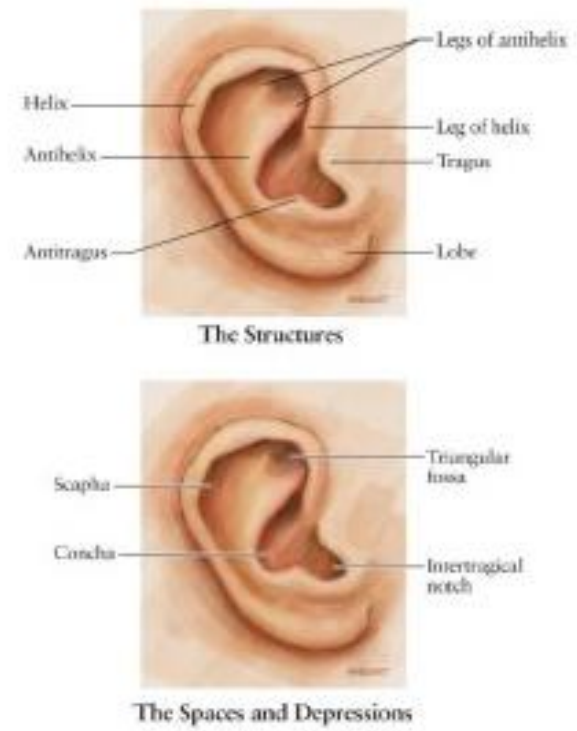

Fig. 1.1 Shows structures, spaces and depressions in ear

\section{SYSTEM OVERVIEW}

In Biometric, Ear recognition is the one of the interesting approach to identify the person's identity. Identifying the person from image is difficult. The process for ear recognition approach with various methods is shown in Fig 2.

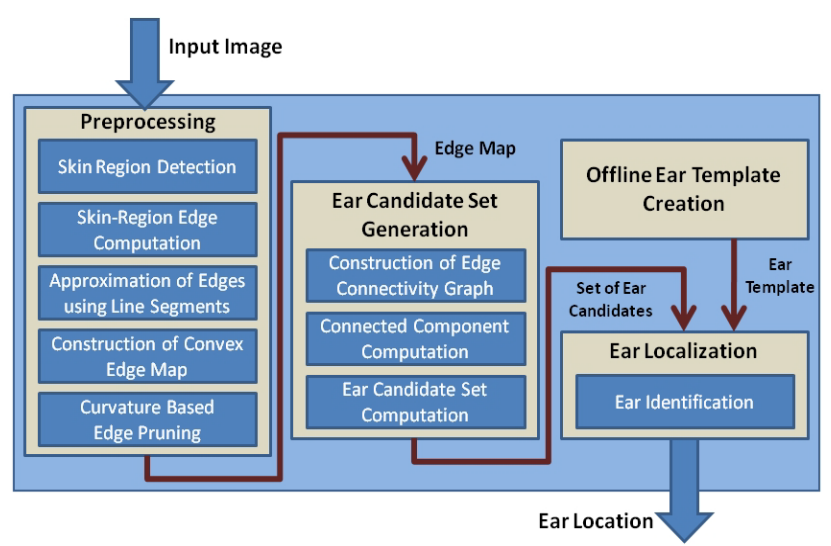

Fig. 2 Flow for Ear Recognition

In initial stage gather the " $n$ " number of ear images respected to the person details and stored in the database by using scanning, gray scale conversion method, median filter and canny edge detection methods. Image acquisition is the main step to capture and position the image in a proper way. Initially scan the image and convert into gray scale image. Next remove the noise in image and find mean vale of image. Next by using canny edge detection mechanism recognize the inner and outer edges they are stored in the database. To recognize the input image, those image edges are identified and treated as " $\mathrm{k}$ ". Using $\mathrm{K}$ Means method match the edge image with database images and find the nearest image to the given input image find the exact image with that process is successfully recognize the person details and says authenticated. Canny edge detection algorithm runs in five steps: Smoothing, Finding gradients, Non-maximum suppression, Double thresholding and Edge tracking by hysteresis. KNN method used to find the exact image to the given image with respect to the person authentication. KNN is related to clustering mechanism. Here, the given image acts as centroid and perform the matching method finding nearest neighbor mechanism and gives the proper recognition.

\subsection{Scanning Ear Image}

This is the initial step to san the given image. In this we are scanning the eye image and storing that image in our database for further purpose. It is treated as original image.

\subsection{RGB Image Converting To Gray Scale Format}

The RGB encoding of pure red is $(255,0,0)$, pure green $(0,255,0)$ and pure blue is $(0,0,255)$.In all RGB encodings, the first value is the amount of red, the second value is the amount of green and the third value is the amount of blue. The range of the three numbers is starting from 0 to ending 255. Gray scale images are in black, white, and all the shades of gray are available. The RGB encoding of any gray values is a set of three equal numbers, i.e., $(\mathrm{x}, \mathrm{x}, \mathrm{x})$, where $\mathrm{x}$ is some integer between 0 and 255. Next step is to converts the full-color image to an 8-bitrepresentation. This reduces space complexity, making further evaluations faster without losing any reliability.

\subsection{Canny Edge Detection Algorithm}

The canny edge detection method derives ear's inner and outer layer edges from the captured image. This gives the effectual edges of ear. So, using this mechanism we can find the exact ear edge boundaries to identify the image and also to authentication will be easy. Canny edge detection algorithm runs in five steps: Smoothing, Finding gradients, Non-maximum suppression, Double thresholding and Edge tracking by hysteresis. They are explained below

Canny edge detection method Smoothing Finding gradients Non-maximum suppression Double thresholding Edge tracking by hysteresis

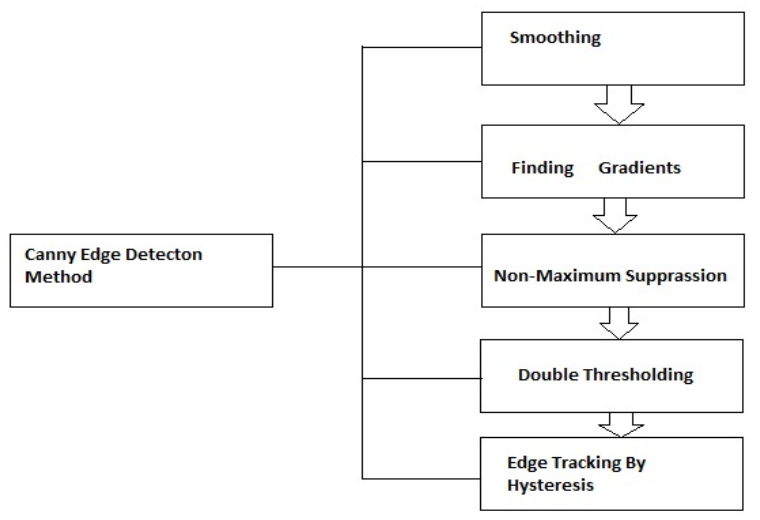

Fig. 3 Canny edge detection process flow representation 


\subsection{Smoothing}

Initially image is captured form camera and that image is having some amount of noise data. So in the early stage only the image is smoothed by applying a Gaussian filter.

\subsection{Finding gradients}

Those areas are found by determining gradients of that image. After smoothing process find the gradient points, which determine each pixel by applying Sobel-operator. Primary step is to estimate the gradient in the $\mathrm{x}$ - and $\mathrm{y}$ direction correspondingly by applying the kernels. The gradient magnitudes are also call as edge strengths, can then be determined as Euclidean distance measured by applying the law of Pythagoras as shown in Equation (1)

a. Sometimes it is easy by applying Manhattan distance measure shown in Equation.

$$
\mathbf{G}=\sqrt{\mathbf{G}_{x}{ }^{2}+\mathbf{G}_{y}{ }^{2}}
$$

Where, $\mathrm{Gx}=$ gradients in the $\mathrm{x}$-direction and Gy are the gradients in the $y$-directions respectively.

Gradient magnitude determines edges with respect to an image clearly. Though, the edges are typically broad and do not indicate precisely where the edges are. The direction of the edges must be determined and stored as shown in Equation (2).

$$
\Theta=\operatorname{atan} 2\left(\mathbf{G}_{y}, \mathbf{G}_{x}\right)
$$

\subsection{Non-maximum suppression}

Converting blurred image of gradient magnitudes to sharp. Essentially this is done by preserving all local maxima in the gradient image, and removing unnecessary data in the image as shown in below steps:

1. Round the gradient Direction $\theta$ to nearest coordinate, equivalent to the use of an 8-connected vicinity.

2. Contrast the edge strength of the existing pixel with the edge strength of the pixel in the optimistic and pessimistic gradient direction.

3. If the edge strength is large for current pixel, then conserve value of the edge strength and if not stem the value.

\subsection{Double Thresholding}

The edge pixels lasting after the non-maximum suppression step are striking with their strength pixel-bypixel. Those pixels will possibly be true edges in the image, but some may cause noise or color variations for graphic due to rough surfaces. To differentiate these pixels, use threshold values then only strongest edge value will conserved. These edge pixels are strong when it has high threshold and weak when they have low threshold.

\subsection{Edge tracking by hysteresis}

Edge tracking can be determined by BLOB-analysis (Binary Large Object). Using 8-connected neighborhood it connect to BLOB's. BLOB's should contains at least one strong edge pixel is then preserved, while other BLOB ${ }^{\text {ee }} \mathrm{S}$ are hidden.

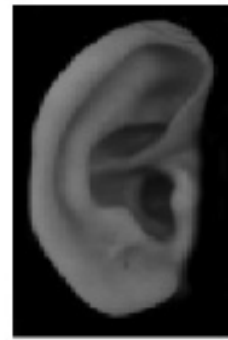

(i)

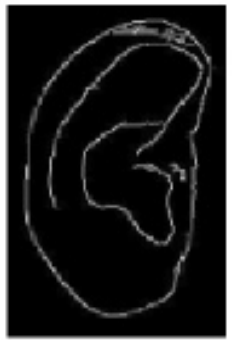

(ii)
Fig 4 (i) sample image (ii) edge detected image

\section{KNN ALGORITHM FOR EAR DETECTION MATCHING EDGE IMAGES}

Identifying the boundaries in the image we find the edges as shown in earlier. To match the edge image with other images in database $\mathrm{KNN}$ is one of the simple methods to solve the well-known clustering problem. The main idea is to define $\mathrm{k}$ centroids from this it is to find the nearest distance image and match that with the required values and gives output respectively. Initially the input image is having edges and that should match with database images. It finds the nearest neighbor image which is relatively suitable to the given image and it gathers all those images. From that a loop is generated and continued till the image found exactly to given image.

\section{APPLICATIONS}

As the most recent publications on 2D and 3D ear recognition show, the main application of this technique is personal identification in unconstrained environments.

This includes applications for smart surveillance, such as in [84] but also the forensic identification of perpetrators on CCTV images or for border control systems. Traditionally these application fields are part of face recognition systems but as the ear is located next to the face, it can provide valuable additional information to supplement the facial images.

Multi modal ear and face recognition systems can serve as a means of achieving pose invariance and more robustness against occlusion in unconstrained environments. In most public venues surveillance cameras are located overhead in order to capture as many persons as possible and to protect them from vandalism. In addition, most of the persons will not look straight into the camera, so in most cases no frontal images of the persons will be available. This fact poses serious problems to biometric systems, using facial features for identification. If the face is not 
visible from a frontal angle, the ear can serve as a valuable additional characteristic in these scenarios. Because of the physical proximity of the face and the ear, there are also many possibilities for the biometric fusion of these two modalities. Face and ear images can be fused on the feature level, on the template level and on the score level. Against the background 25of this application, there are some unsolved challenges, which should be addressed by future research in these fields.

\section{CONCLUSION}

In ear recognition, the inner and outer layer edges give effective shape of ear to recognize a person. Using traditional methods cause the problem of ear inheritance and loss of texture. Canny edge detection, effectively improves the ear positioning accuracy and KNN technique is to match the edges with other image edges. It increases the accuracy and efficient speed of ear recognition with less complexity and less space of memory. If this approach is used along with face recognition process then it shows more security. Because if the image of a person is having only one side part of his face then authentication is not possible. By using, ear part from the image authentication process done along with one side of face part. It shows speed and accurate authentication process. It is low cost and performance is better.

\section{ACKNOWLEDGMENTS}

We are sincerely thankful to Prof. S. B. Waykar to enlighten us with their insightful knowledge which helped us to succeed in completing the project.

\section{REFERENCES}

[1] Anika Pug, Christoph Busch." Ear Biometrics: A Survey of Detection, Feature Extraction and Recognition Methods". IET Biometrics, pp.1-35. July 2, 2012.

[2] Ajay Kumar n and Chenye Wu," Automated human identification using ear imaging". Automated human identification using ear imaging, Pattern Recognition, Elsevier. pp. 1-13, 2011.

[3] S.M.S. Islam, R. Davies, A.S. Mian, and M. Bennamoun," A Fast and Fully Automatic Ear Recognition Approach Based on 3D Local SurfaceFeatures". Springer -Verlag Berlin Heidelberg. pp. 10811092. 2008.

[4] Lu lu, zhang xiaoxun, zhao youdong and jia yunde, "ear recognition based on statistical shape model".International conference on innovative computing, information and control (icicic), ieee, 2006.

[5] Ping yan and kevin w. bowyer, "biometric recognition using $3 \mathrm{~d}$ ear shape", ieee transactions on pattern analysis and machine intelligence, vol. 29, no. 8. pp. 1-12. August 2007.

[6] Li Yuan, Zhi-chun Mu," Ear Recognition based on 2D Images", IEEE, 2007. 